\title{
Using ARToolkit to Prototype Future Entertainment Scenarios
}

\author{
Adrian Woolard ${ }^{1}$, Vali Lalioti ${ }^{2}$, Nicholas Hedley ${ }^{3}$, Joey Julien ${ }^{1}$, Matt Hammond ${ }^{2}$, Neil Carrigan ${ }^{1}$ \\ ${ }^{1} B B C$ Creative $R \& D$, $U K$ \\ ${ }^{2} B B C$ Research \& Development, $U K$ \\ vali.lalioti@rd.bbc.co.uk \\ ${ }^{3}$ ARToolworks, Inc, USA \\ nix@u.washington.edu
}

\begin{abstract}
This demonstration poster illustrates the potential application of augmented reality (AR) in creation of future interactive entertainment experiences. It describes the production of prototypes using ARToolkit that use physically manipulated AR within television broadcast production and in home entertainment for children.
\end{abstract}

\section{Background}

The majority of the entertainment industry has yet to take full advantage of the potential of Augmented Reality (AR) technology compared with military and industrial applications [1]. Reasons for this include the practical requirements and tight schedule of television production allowing only minimal experimentation with new technologies; and the fact AR is in its infancy in terms of providing robust real-time systems that can be confidently used by production staff.

The BBC has recently begun to evaluate the potential of AR in future broadcast and online services [2]. The work has produced a number of prototypes that utilize the ARToolkit [3]. In assessing the functionality of AR to physically manipulate virtual objects, it is important to understand the importance of the narrative or editorial content in producing a compelling story / documentary. ARToolkit allows easy creation of prototypes allowed rapid investigation of the viewer or user experience.

\section{Adapting ARToolkit for Broadcast Studios}

Within broadcasting, areas such as sports have used realtime augmentation of the video to display 2D match statistics, critical information related to the play such as offside or the location of the ice puck and advertising banners [4]. More advanced technologies, used in studio production, are 'virtual studios' which merge people and real objects within a fully synthetic 3D set. Virtual studios typically require a purpose-build space covered with chroma-keying material (blue, green or retro-reflective) and use robust and highly accurate camera tracking systems [5], [6]. The cumbersome set-up and calibration process, in conjunction with high cost of physical studio space and camera tracking systems make its ad-hoc use difficult in a conventional studio or outdoors. In the above systems, interaction between real and virtual components is limited to pre-scripted animations manually triggered by the director resulting in minimal interactions between the real and virtual elements. Also, the interaction between virtual elements has not been attempted and is considered difficult to achieve, as majority of systems use separate devices to track each pattern.

The 'War Board' prototype developed with ARToolkit explored the use of AR for analysis of a fictional military conflict enabling a presenter to physically manipulate virtual objects in a standard television studio. Several extensions were needed to allow this type of tracking with a broadcast camera. For example, dealing with interlaced images, zooming of camera lens, linking to a proprietary high-end rendering engine, ability to merge virtual objects both in front and behind real elements and chroma-keying patterns that can be also recognized by the visual tracking algorithms of the ARToolkit.

A 3D landscape was linked to an AR tracked pattern placed on a table surface. The presenter could then position military forces represented by $3 \mathrm{D}$ tanks or incorporate live video streams displayed via virtual walls, using other AR trained patterns, in key areas of the landscape. Examples are shown in Figure 1.

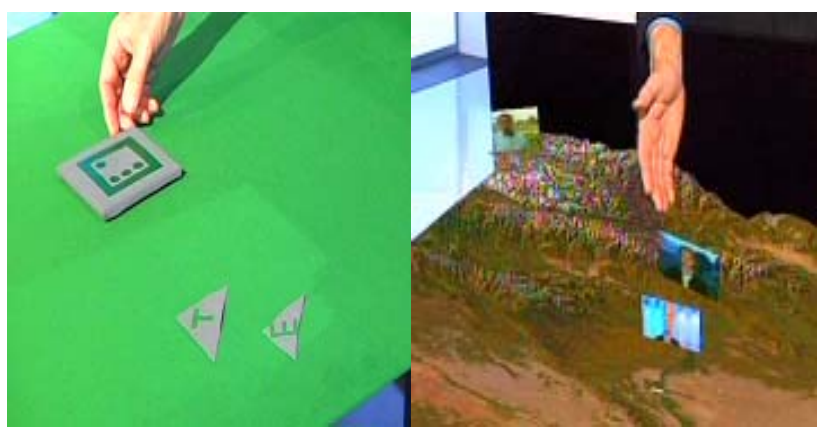

Figure 1 Camera image of tracked AR pattern and live video inserts of correspondents within the landscape

Occlusion and the effects of shadows and lights on object tracking were identified as robustness issues with ARToolkit in the studio environment. However, the initial results were disseminated to $\mathrm{BBC}$ production staff and reviewed favourably in comparison with existing technologies. ARToolkit allowed fast assessment of the 
potential use of this type of AR interface in a broadcast environment across many different genres.

\section{Adding Interactive Narrative Content for Entertainment}

The Magic Book [7] allowed users to interact with nonimmersive AR interfaces demonstrating a potentially new and original method for the creation of entertainment that merges physical objects, such as a book, with the sophistication of current computer games. Marks [8] work was a further example of potential of enhanced reality and previous BBC work with 3D virtual worlds [9] suggested great potential for original and compelling interactive stories.

The research was based on story elements of a children's drama television series aimed at 8 to 12 year olds. The design of the interactive elements used principles of computer game design [10] defining; aims, ambitions; interactions and games spaces. For example, the overall 'aim' was to manage a team of virtual characters trying to save a rare creature in a futuristic setting and the 'emotional game-space' was engaging with the story and a greater understanding of the natural world.

The work produced five mini-games using a variety of prototypes including ARToolkit applications, a web application called Pulse Veepers ${ }^{\mathrm{TM}}$ [11] and a human "storyteller". The facilitator provided guidance on elements of the game functionality that could not be authored using ARToolkit. The mini-games included a variety of goal-based tasks to be solved including; character selection (Figure 2), exploration of a virtual world and a 'race-against-the-clock' construction of a 3D structure.

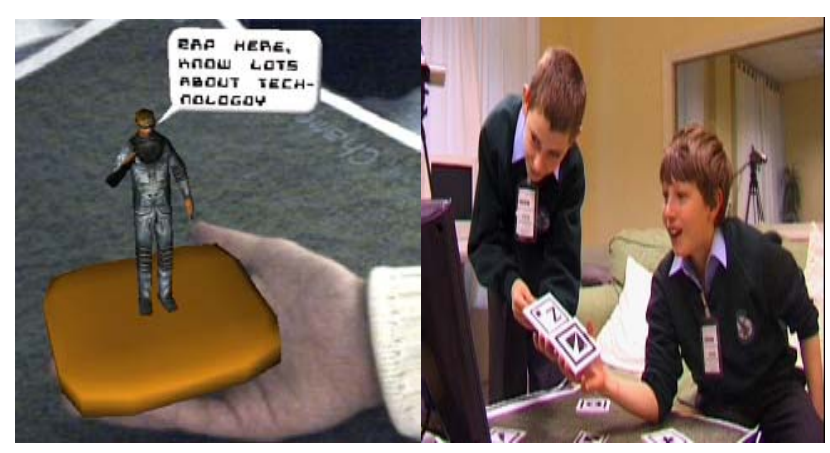

Figure 2 Examples of AR enhanced content and Children playing in user lab

Initial qualitative and observation data suggested that the introduction of narrative content, coupled to an AR interface, produced positive responses from 12 users. They were able to recognize and manipulate the different virtual characters and objects displayed with a high tolerance for current tracking limitations. These resulted from shadows, occlusion and mis-handling of patterns. All users liked the challenges of each mini-game though there was general agreement about desire for greater complexity and elements of skill in the mini-games, currently limited by the lack of authoring tools available.

\section{Discussion}

The primary motivation in this work was to understand how AR enhanced editorial content to improve the viewer or user experience. The use of ARToolkit to develop rapid prototyping of content and interactive $A R$ applications allowed great flexibility to engage and stimulate new scenarios from both creative staff and children. Key users such as the presenter, director and producer were able to make an immediate connection between the mode of interaction presented and their own content and editorial ideas, making the use of the underlying technology transparent to the user.

From a technical perspective, the evaluation highlighted the need for more robust tracking to minimize the impact of environmental factors such as lighting. Further development is required into authoring tools of dynamic and interactive AR content to enable more complex models of interaction. These tools should be appropriate for non-programmers and integrate into existing content creation 'pipelines'. Access to the types of functionality currently available on other platforms such as web and computer games may well see development of more complex and engaging challenges for user experiences.

\section{References}

[1] Azuma, R.T., et al. "Recent Advances in Augmented Reality”. IEEE Computers \& Graphics, Nov./Dec. 2001:34-47

[2] Woolard, A., et al, "Case Studies in Application of Augmented Reality in Future Media Production", In Proc. ISMAR 2003, (in publication), Japan 2003

[3] http://www.hitl.washington.edu/artoolkit

[4] Cavallaro, R., "The FoxTrax Hockey Puck Tracking System," IEEE Computer Graphics \& Applications, 17(2), Mar/Apr 1997: 6-12

[5] Thomas, G., "Prompting Guide for Chroma-Keying", US Patent No5,886,747.

[6] Gibbs, S., et al., "Virtual Studios: State of the Art", IEEE MultiMedia magazine, January-March, 1998.

[7] Billinghurst, M., Kato, H., Poupyrev, I., "The Magic Book-Moving Seamlessly between Reality \& Virtuality”, IEEE Computers Graphics \& Applications, 21(3), May/Jun 2001: 2-4

[8] Marks, R., "Enhanced Reality: A new frontier in computing entertainment", Sketch paper, Siggraph 2001 Proceedings, 2001.

[9] Fletcher, J., "Simutainment: A factual TV documentary as a 3D interactive experience", Proc. IBC 2002, Sept 2002.

[10] Adams, E, "Game Design Philosophy", http://www.designersnotebook.com/Design_Philosophy/

[11] http://www.pulse3d.com/pulse/veepers.html 